\title{
A CONVERGENCE THEOREM FOR CONTINUED FRACTIONS*
}

\author{
BY
}

\author{
W. T. SCOTT AND H. S. WALL
}

1. Introduction. This paper is concerned with the question of convergence of continued fractions of the form

$$
\frac{1}{1}+\frac{a_{2}}{1}+\frac{a_{3}}{1}+\frac{a_{4}}{1}+\cdots
$$

in which the $a_{n}$ are complex numbers. Our investigation began with a comparison of the infinite series

$$
\frac{A_{1}}{B_{1}}+\left(\frac{A_{2}}{B_{2}}-\frac{A_{1}}{B_{1}}\right)+\left(\frac{A_{3}}{B_{3}}-\frac{A_{2}}{B_{2}}\right)+\cdots,
$$

equivalent to the sequence of approximants of the continued fraction, with a convergent positive term series $\sum p_{n}$. It was found that if $r_{n}=p_{n+1} / p_{n}$, and

$$
r_{n}\left|1+a_{n}+a_{n+1}\right| \geqq r_{n} r_{n-2}\left|a_{n}\right|+\left|a_{n+1}\right|,
$$

$n=1,2,3, \cdots, r_{0}=r_{-1}=a_{1}=0$, then the series $\sum p_{n}$ is a majorant for (1.2), so that the continued fraction converges.

Later, in proving that the Pringsheim convergence theorem can be obtained from the above by specializing the series $\sum p_{n}$, it was discovered that the inequalities (1.3) have a second interpretation. In fact, if $a_{n} \neq 0$, and

$$
y_{0}+\frac{x_{1}}{y_{1}}+\frac{x_{2}}{y_{2}}+\frac{x_{3}}{y_{3}}+\cdots
$$

denotes the even (odd) "contraction" of (1.1), then the inequalities (1.3) for odd (even) values of $n$ may be written in the form

$$
\left|c_{1} y_{1}\right| \geqq 1, \quad\left|c_{n} y_{n}\right| \geqq\left|c_{n-1} c_{n} y_{n}\right|+1,
$$

$n=2,3,4, \cdots$, where $c_{n} \neq 0$. But these are precisely the Pringsheim relations $\dagger$ for the continued fraction (1.4). Hence if the inequalities (1.3) hold for some $r_{n}>0$ (not necessarily related to a convergent series $\sum p_{n}$ ), the

* Presented to the Society, September 8, 1939; received by the editors May 26, 1939, and, in revised form, October 27, 1939.

† O. Perron, Die Lehre von den Kettenbrüchen (referred to hereafter as "Perron"), 2d edition, Leipzig and Berlin, 1929, pp. 257-258, formulas (5). 
sequences of even and odd approximants of (1.1) have limits, finite or infinite, and the limit of the even (odd) approximants is finite if inequality holds in (1.3) for an odd (even) index. Thus the relations (1.3) have two interpretations, and therefore are extremely important instruments in the convergence theory.

One of the most important results obtained in the paper is the "parabola theorem," namely, that if the elements $a_{n}$ of (1.1) lie within or upon the parabola $|z|-\Re(z)=1 / 2$, then the continued fraction converges if and only if some $a_{n}$ is 0 , or the series $\sum\left|b_{n}\right|$ diverges, where $b_{1}=1, a_{n}=1 / b_{n-1} b_{n}$, $n=2,3,4, \cdots$. In the first announcement of the theorem, ${ }^{*}$ the $a_{n}$ were restricted to lie in a closed bounded region entirely within the parabola, a result which was obtained using $r_{n}=r<1$ in (1.3). Using the second interpretation of the inequalities, we were able later to remove the restriction requiring the $a_{n}$ to lie in the interior of the parabola, and to replace the boundedness condition by the condition on the series $\sum\left|b_{n}\right|$.

The parabola cannot be replaced by a "better" curve symmetric with respect to the real axis. We thus characterize completely all regions in the complex plane, symmetric with respect to the real axis, in which the elements $a_{n}$ may vary independently while the continued fraction remains convergent. We have therefore, in a certain sense, extended as far as possible the theorem given by Worpitzky $\dagger$ three-quarters of a century ago, namely, that (1.1) converges if $\left|a_{n}\right| \leqq 1 / 4, n=2,3,4, \cdots$.

Other results obtained include: a notable improvement in the Pringsheim theorem on uniform convergence (Theorem B), the "triangle theorem" (Theorem G), some results on the problem of Szász (\$6), and the theorem that if (1.3) holds and the $a_{n}$ are bounded, then (1.1) converges (Theorem E).

The methods used throughout the paper are elementary in character, and are based strictly upon the continued fraction algorithm.

2. The first interpretation of the fundamental inequalities. The basic result of this paper is embodied in the theorem which follows.

TheOREM A. Let (1.1) be a continued fraction with complex elements $a_{n}$, and nth approximant $A_{n} / B_{n}$. Suppose that for some nonnegative numbers $r_{n}$ the inequalities (1.3) hold. Then $B_{n} \neq 0, n=1,2,3, \cdots$, and the series $1+\sum r_{1} r_{2} \cdots r_{n}$ is a majorant for the series (1.2). The continued fraction converges if some $a_{n}$ is 0 , or if the series $1+\sum r_{1} r_{2} \cdots r_{n}$ converges. The sum of the latter series is an upper bound for the absolute value of the continued fraction.

Proof. By (1.3) we have $B_{2}=1+a_{2} \neq 0, B_{3}=1+a_{2}+a_{3} \neq 0$; and if we

* Bulletin of the American Mathematical Society, abstract 45-7-277.

$\dagger$ Worpitzky, Jahresbericht, Friedrichs-Gymnasium und Realschule, Berlin, 1865, pp. 3-39. Independent proofs of this theorem were given later by Pringsheim and by Van Vleck. 
put $c_{n}=a_{n+1} B_{n-1} / B_{n+1}, n=1,2,3, \cdots$, we have $\left|c_{1}\right|=\left|a_{2} /\left(1+a_{2}\right)\right| \leqq r_{1}$, $\left|c_{2}\right|=\left|a_{3} /\left(1+a_{2}+a_{3}\right)\right| \leqq r_{2}$. Let us assume that $B_{n+1} \neq 0,\left|c_{n}\right| \leqq r_{n}$, for $n=1,2,3, \cdots, k, k \geqq 2$, and prove that the same holds for $n=k+1$, and hence for all $n$. Inasmuch as

$$
\left|\frac{A_{n+1}}{B_{n+1}}-\frac{A_{n}}{B_{n}}\right|=\left|c_{1} c_{2} \cdots c_{n}\right|,
$$

it will follow that $1+\sum r_{1} r_{2} \cdots r_{n}$ is a majorant for the series (1.2), so that the continued fraction converges if $1+\sum r_{1} r_{2} \cdots r_{n}$ converges. Since $B_{n} \neq 0$, for all $n$, the continued fraction converges if some $a_{n}$ is 0 .

From the recursion relation $B_{n}=B_{n-1}+a_{n} B_{n-2}, n \geqq 2$, we easily obtain the formula

$$
B_{k+2}=\left(1+a_{k+1}+a_{k+2}\right) B_{k}-a_{k} a_{k+1} B_{k-2},
$$

$k=2,3,4, \cdots$. If $a_{k+2} \neq 0$, so that $r_{k+1}>0$, then we have

$$
\frac{B_{k+2}}{a_{k+2} B_{k}}=\frac{1+a_{k+1}+a_{k+2}}{a_{k+2}}-\frac{a_{k+1}}{a_{k+2}} \frac{a_{k} B_{k-2}}{B_{k}},
$$

or, by (1.3) and our assumption,

$$
\left|\frac{B_{k+2}}{a_{k+2} B_{k}}\right| \geqq\left|\frac{1+a_{k+1}+a_{k+2}}{a_{k+2}}\right|-\left|\frac{a_{k+1}}{a_{k+2}}\right| r_{k-1} \geqq \frac{1}{r_{k+1}}>0 .
$$

Hence $B_{k+2} \neq 0$, and $\left|c_{k+1}\right| \leqq r_{k+1}$. On the other hand, if $a_{k+2}=0$, it follows from the fundamental recursion formula that $B_{k+2}=B_{k+1} \neq 0$, and $\left|c_{k+1}\right|=0 \leqq r_{k+1}$. This completes the induction and the proof of Theorem $A$.

Since Theorem A is simply a comparison theorem for the series (1.2), we have at once by the Weierstrass "M-test," the following theorem on uniform convergence of continued fractions.

THEOREM 2.1. If in the continued fraction (1.1) of Theorem A, the elements $a_{n}$ are functions of any variables over a domain $D$ in which the inequalities (1.3) hold, with the $r_{n}$ independent of the variables, and if the series $1+\sum r_{1} r_{2} \cdots r_{n}$ converges, then the continued fraction converges uniformly over $D$.

If in Theorem A we use the series

$$
\frac{A_{k+1}}{B_{k+1}}+\left(\frac{A_{k+2}}{B_{k+2}}-\frac{A_{k+1}}{B_{k+1}}\right)+\left(\frac{A_{k+3}}{B_{k+3}}-\frac{A_{k+2}}{B_{k+2}}\right)+\cdots
$$

instead of the series (1.2) we get by the same methods the more general result: 
THEOREM $\mathrm{A}^{\prime}$. If there exist an index $k \geqq 0$, and numbers $r_{n} \geqq 0, n=k+1$, $k+2, k+3, \cdots$, such that $A_{k+1}, B_{k+2}, B_{k+3} \neq 0$ and

$$
\begin{gathered}
r_{k+1}\left|B_{k+2} A_{k+1}\right| \geqq\left|a_{2} a_{3} \cdots a_{k+2}\right|, \quad r_{k+2}\left|B_{k+3}\right| \geqq\left|a_{k+3} B_{k+1}\right|, \\
r_{n}\left|1+a_{n}+a_{n+1}\right| \geqq r_{n} r_{n-2}\left|a_{n}\right|+\left|a_{n+2}\right|,
\end{gathered}
$$

$n=k+3, k+4, k+5, \cdots$, then the series

$$
\left|\frac{A_{k+1}}{B_{k+1}}\right|\left(1+\sum_{n=k+1}^{\infty} r_{k+1} r_{k+2} \cdots r_{n}\right)
$$

is a majorant for the series (2.2). The continued fraction converges if some $a_{n}$ is 0 for $n \geqq k+2$, or if the series (2.3) converges.

3. Relation of Theorem A to other convergence criteria. We now prove a second theorem.

Theorem B. Let $p_{1}, p_{2}, p_{3}, \cdots$ be real and positive, $p_{1}>1$. Then the continued fraction (1.1) in which the elements $a_{n}$ are functions of any variables converges uniformly over the domain characterized by the inequalities

$$
\left|a_{n}\right| \leqq\left(p_{n}-1\right) / p_{n} p_{n-1}, \quad n=2,3,4, \cdots \text {. }
$$

The quantity

$$
1+\frac{p_{1}}{p_{1}-1}\left\{\frac{1}{p_{1}}-\frac{1}{1+\sum_{1}^{\infty}\left(p_{1}-1\right)\left(p_{2}-1\right) \cdots\left(p_{n}-1\right)}\right\}
$$

is an upper bound for the absolute value of the continued fraction over the domain, and is actually attained if $a_{n}=\left(1-p_{n}\right) / p_{n} p_{n-1}, n=2,3,4, \cdots$.

This theorem includes the general Pringsheim criterion. $\dagger$ However, our theorem goes farther and gives an upper bound for the absolute value of the continued fraction. The greatest improvement is in respect to the question of uniform convergence. Pringsheim required that the series $\sum\left(p_{1}-1\right)\left(p_{2}-1\right) \cdots\left(p_{n}-1\right)$ be divergent in order to obtain uniform convergence of the continued fraction. $\ddagger$

Proof of Theorem B. Let $t_{n}=\left(p_{n}-1\right) p_{n} p_{n-1}$, and form the continued fraction

$$
1 / 1-t_{2} / 1-t_{3} / 1-\cdots \cdot
$$

Let $G_{n} / H_{n}$ be the $n$th convergent of (3.3) and put

$$
H_{n}^{*}=p_{1} p_{2} \cdots p_{n} H_{n}, \quad n=1,2,3, \cdots, \quad H_{0}^{*}=1,
$$

$\dagger$ Perron, p. 258, Theorem 26. It is to be noted that there is no loss in generality in assuming $p_{1}>1$ in that theorem.

$\ddagger$ Perron, p. 262, Theorem 30. 
so that $H_{n}^{*}=p_{n} H_{n-1}^{*}-p_{n} p_{n-1} t_{n} H_{n-2}^{*}, n=2,3,4, \cdots$, and

$$
H_{n}^{*}-H_{n-1}^{*}=\left(p_{n}-1\right)\left(H_{n-1}^{*}-H_{n-2}^{*}\right)=\left(p_{1}-1\right)\left(p_{2}-1\right) \cdots\left(p_{n}-1\right),
$$

$n=1,2,3, \cdots$. Thus $H_{n}^{*} \geqq 1$, and consequently $H_{n}>0$ for all $n$.

We take for $r_{n}$ in Theorem A the quantity

$$
r_{n}=t_{n+1} H_{n-1} / H_{n+1}, \quad n=1,2,3, \cdots .
$$

Inasmuch as $H_{n+1}=\left(1-t_{n}-t_{n+1}\right) H_{n-1}-t_{n} t_{n-1} H_{n-3}$, we readily verify that $r_{n}\left(1-t_{n}-t_{n+1}\right)=r_{n} r_{n-2} t_{n}+t_{n+1}, n=3,4,5, \cdots,\left(r_{0}=r_{-1}=t_{1}=0\right)$. Hence it follows from (3.1) and the definition of $t_{n}$ that the elements of the continued fraction satisfy the inequalities of Theorem A with the values of the $r_{n}$ which we have determined.

Since

$$
r_{1} r_{2} \cdots r_{n}=\frac{p_{1}}{p_{1}-1}\left[\frac{1}{H_{n}^{*}}-\frac{1}{H_{n+1}^{*}}\right]
$$

it follows that the series $1+\sum r_{1} r_{2} \cdots r_{n}$ converges; and since the $r_{n}$ are independent of the variables, it follows by Theorem 2.1 that the continued fraction converges uniformly over the domain of the variables.

The quantity (3.2) is simply the sum of the majorant series, and is therefore an upper bound for the absolute value of the continued fraction. It is attained by the continued fraction when $a_{n}=-t_{n}$.

This completes the proof of Theorem $\mathrm{B}$.

By specializing the $p_{n}$ in Theorem B the familiar special Pringsheim criteria $\dagger$ may be obtained, with the attendant upper bounds. For example, setting $p_{n}=2$ we obtain this theorem:

THEOREM 3.1. (Worpitzky.) The continued fraction (1.1) converges if $\left|a_{n}\right| \leqq 1 / 4, n=2,3,4, \cdots$.

In this case the upper bound for the absolute value of the continued fraction is 2 , and if $a_{n}=-1 / 4$, this value is attained.

The importance of Theorem $\mathrm{B}$ is illustrated by the fact that the proof of the following theorem of Van Vleck as given by Perron $\ddagger$ can now be considerably shortened.

Theorem 3.2. (Van Vleck.) Let $s_{1}, s_{2}, s_{3}, \cdots$ be real numbers, and $t_{1}, t_{2}, t_{3}, \cdots$ complex numbers, such that

$$
\begin{array}{cc}
0<s_{1}<1, \quad 0 \leqq s_{n}<1, & n=2,3,4, \cdots, \\
\left|t_{n}\right| \leqq 1, & n=1,2,3, \cdots ;
\end{array}
$$

† Perron, pp. 259-260.

$\ddagger$ Perron, pp. 262-264. 
and let $x$ be a complex variable. Then the continued fraction

$$
\frac{s_{1} t_{1} x^{n_{1}}}{1}+\frac{s_{2}\left(1-s_{1}\right) t_{2} x^{n_{2}}}{1}+\frac{s_{3}\left(1-s_{2}\right) t_{3} x^{n_{3}}}{1}+\cdots
$$

( $n_{i}$ positive integers) converges uniformly for $|x| \leqq 1$. The modulus of the analytic function which the continued fraction represents cannot exceed

$$
s_{1}\left|t_{1} x^{n_{2}}\right|\left(1+\frac{1-s_{1}}{s_{1}}\left[1-\frac{1}{1+\left(1-s_{1}\right) \sum_{1}^{\infty} \frac{s_{1} s_{2} \cdots s_{n}}{\left(1-s_{1}\right)\left(1-s_{2}\right) \cdots\left(1-s_{n}\right)}}\right]\right) .
$$

Proof. Take $p_{n}=1 /\left(1-s_{n}\right), n=1,2,3, \cdots$, in Theorem B.

As an illustration of the last theorem, the function $x /(\operatorname{arc} \tan x)$ has the continued fraction expansion*

$$
1+\frac{\frac{x^{2}}{1 \cdot 3}}{1}+\frac{\frac{4 x^{2}}{3 \cdot 5}}{1}+\frac{\frac{9 x^{2}}{5 \cdot 7}}{1}+\cdots
$$

to which Theorem 3.2 is applicable with $s_{n}=n /(2 n+1)$. We find that the modulus of the function does not exceed $1+\left|x^{2}\right|$ if $|x| \leqq 1$.

In the proof of the next theorem we use for the first time the second interpretation of the fundamental inequalities, which was mentioned in the introduction.

THEOREM C. The continued fraction of Theorem A converges at least in the wider sense if

$$
\lim _{n=\infty} \inf \left(r_{1} r_{2} r_{3} \cdots r_{n}\right)=0
$$

and converges to a finite value if (3.4) holds and actual inequality holds at least once in (1.3).

Proof. We need only consider the case where $a_{n} \neq 0, n=2,3,4, \cdots$, since Theorem A disposes of the case where some $a_{n}$ is 0 . If $a_{n} \neq 0$ for all $n$, it is clear that $r_{n}>0$ for all $n$.

Consider the continued fractions

$$
\begin{gathered}
\frac{1}{1+a_{2}}-\frac{a_{2} a_{3}}{1+a_{3}+a_{4}}-\frac{a_{4} a_{5}}{1+a_{5}+a_{6}}-\cdots, \\
1-\frac{a_{2}}{1+a_{2}+a_{3}}-\frac{a_{3} a_{4}}{1+a_{4}+a_{5}}-\frac{a_{5} a_{6}}{1+a_{6}+a_{7}}-\cdots .
\end{gathered}
$$

* Perron, p. 351, formula (17) (in slightly different form). 
The approximants of (3.5) are the even approximants* of (1.1), while those of (3.6) are the odd approximants of (1.1). We now write the inequalities (1.3) in the form

$$
\begin{array}{rl}
\frac{r_{1}}{\left|a_{2}\right|}\left|1+a_{2}\right| \geqq 1, & \geqq a_{2 n+1}+a_{2 n+2}\left|\geqq \frac{r_{2 n+1}}{\left|a_{2 n+2}\right|} \frac{r_{2 n-1}}{\left|a_{2 n}\right|}\right| a_{2 n} a_{2 n+1} \mid+1 ; \\
\frac{r_{2 n+1}}{\left|a_{2 n+2}\right|} \mid 1+a_{2 n} & 1+a_{2}+a_{3} \mid \geqq 1, \\
\left|a_{3}\right| & \geqq \frac{r_{2 n+2}}{\left|a_{2 n+3}\right|} \frac{r_{2 n}}{\left|a_{2 n+1}\right|}\left|a_{2 n+1} a_{2 n+2}\right|+1,
\end{array}
$$

$n=1,2,3, \cdots$. But the conditions (3.7) constitute a Pringsheim test for (3.5) provided inequality holds at least once; $\dagger$ and the same is true for conditions (3.8) and the continued fraction (3.6). It should be noted that the continued fractions (3.5), (3.6) may diverge to $\infty$ if at every stage in the conditions (3.7), (3.8) equality holds. In any case the $\operatorname{limits} \lim _{n=\infty}\left(A_{2 n} / B_{2 n}\right)=L_{0}$, $\lim _{n=\infty}\left(A_{2 n-1} / B_{2 n-1}\right)=L_{1}$ exist, finite or infinite.

But by Theorem A,

$$
\left|\frac{A_{n+1}}{B_{n+1}}-\frac{A_{n}}{B_{n}}\right| \leqq r_{1} r_{2} \cdots r_{n}
$$

so that by (3.4), $L_{0}=L_{1}$. If inequality holds at least once in (1.3), then this common limit of the two sequences is finite, and the continued fraction converges.

In a recent paper, Leighton $₫$ obtained a convergence theorem for (1.1) by applying the Pringsheim inequalities to (3.7) and (3.8) separately. Since the element $a_{1}$ appearing in his work can have no bearing upon the convergence of the continued fraction, it will be seen that his result may be stated as follows:

Theorem 3.3. (Leighton.) Let§

$$
\begin{gathered}
\left|1+a_{2}\right| \geqq 1, \quad\left|1+a_{2}+a_{3}\right| \geqq 1, \\
\left|1+a_{n}+a_{n+1}\right| \geqq\left|a_{n-1} a_{n}\right|+1,
\end{gathered}
$$

* Perron, p. 201, formulas (7), (8).

† See the second footnote.

‡ Walter Leighton, Duke Mathematical Journal, vol. 4 (1938), pp. 775-778.

$\S$ The proof as given by Leighton would not permit equality in the first two relations, which, however, we allow only when some $a_{n}$ vanishes. 
$n=3,4,5, \cdots$. Then the continued fraction (1.1) converges if some $a_{n}$ vanishes, or if actual inequality holds in the first two relations (3.9) and $\lim \inf \left|a_{n}\right|<\infty$.

Proof. Multiply the inequalities (3.9) by $\left|a_{2}\right|,\left|a_{3}\right|,\left|a_{4}\right|, \cdots$, respectively, and they take the form (1.3) where $r_{n}=\left|a_{n+1}\right|$. Hence if some $a_{n}$ vanishes, the continued fraction converges by Theorem $A$.

If $a_{n} \neq 0, n \geqq 2$, then by hypothesis actual inequality holds in the first two relations (1.3), so that (3.5), (3.6) converge. Moreover, the quantities

$$
g_{1}=\left|B_{2}\right|-\frac{\left|a_{2}\right|}{r_{1}}\left|B_{0}\right|, \quad g_{2}=\left|B_{3}\right|-\frac{\left|a_{3}\right|}{r_{2}}\left|B_{1}\right|
$$

are positive. We then have by (2.1), (1.3), the inequalities

$$
\left\{\left|B_{k+2}\right|-\frac{\left|a_{k+2}\right|}{r_{k+1}}\left|B_{k}\right|\right\} \geqq r_{k-1}\left|a_{k+1}\right|\left\{\left|B_{k}\right|-\frac{\left|a_{k}\right|}{r_{k-1}}\left|B_{k-2}\right|\right\}, \quad k \geqq 2,
$$

and therefore

$$
\begin{aligned}
& \left|B_{2 n+2}\right|-\frac{\left|a_{2 n+2}\right|}{r_{2 n+1}}\left|B_{2 n}\right| \geqq r_{1} r_{3} \cdots r_{2 n-1}\left|a_{3} a_{5} \cdots a_{2 n+1}\right| g_{1}>0, \\
& \left|B_{2 n+3}\right|-\frac{\left|a_{2 n+3}\right|}{r_{2 n+2}}\left|B_{2 n+1}\right| \geqq r_{2} r_{4} \cdots r_{2 n}\left|a_{4} a_{6} \cdots a_{2 n+2}\right| g_{2}>0 .
\end{aligned}
$$

When $r_{n}=\left|a_{n+1}\right|$, these inequalities become

$$
\begin{aligned}
\left|B_{2 n+2}\right|-\left|B_{2 n}\right| & \geqq\left|a_{2} a_{3} \cdots a_{2 n+1}\right| g_{1}, \\
\left|B_{2 n+3}\right|-\left|B_{2 n+1}\right| & \geqq\left|a_{3} a_{4} \cdots a_{2 n+2}\right| g_{2} .
\end{aligned}
$$

Consequently,

$$
\begin{aligned}
& \left|\frac{A_{2 n+2}}{B_{2 n+2}}-\frac{A_{2 n+1}}{B_{2 n+1}}\right| \leqq \frac{\left|a_{2}\right|}{g_{2}} \frac{1}{\left|B_{2 n+2}\right|}\left(\left|\frac{B_{2 n+3}}{B_{2 n+1}}\right|-1\right), \\
& \left|\frac{A_{2 n+1}}{B_{2 n+1}}-\frac{A_{2 n}}{B_{2 n}}\right| \leqq \frac{1}{g_{1}} \frac{1}{\left|B_{2 n+1}\right|}\left(\left|\frac{B_{2 n+2}}{B_{2 n}}\right|-1\right) .
\end{aligned}
$$

Since by (3.11) the sequences $\left|B_{2 n}\right|$ and $\left|B_{2 n-1}\right|$ are strictly monotone increasing, they must have limits, finite or infinite. If either has a finite limit, we see from (3.12), remembering that (3.5), (3.6) both converge, that the continued fraction (1.1) converges. If $\lim \left|B_{2 n}\right|=\lim \left|B_{2 n-1}\right|=\infty$, we have the inequalities

$$
\left|\frac{B_{2 n+2}}{B_{2 n} B_{2 n+1}}\right| \leqq \frac{1}{\left|B_{2 n}\right|}+\frac{\left|a_{2 n+2}\right|}{\left|B_{2 n+1}\right|}, \quad\left|\frac{B_{2 n+3}}{B_{2 n+1} B_{2 n+2}}\right| \leqq \frac{1}{\left|B_{2 n+1}\right|}+\frac{\left|a_{2 n+3}\right|}{\left|B_{2 n+2}\right|},
$$


so that under the hypothesis lim inf $\left|a_{n}\right|<\infty$, the right-hand member of at least one of (3.12) must have the inferior limit 0 , and, therefore, the continued fraction converges in this case also.

Of course, the inequalities (3.10) hold whenever the $r_{n}>0$ exist satisfying (1.3) with inequality for $n=1,2$. We therefore have

$$
\begin{aligned}
\left|\frac{A_{2 n+3}}{B_{2 n+3}}-\frac{A_{2 n+2}}{B_{2 n+2}}\right|= & \frac{\left|a_{2} a_{3} \cdots a_{2 n+3}\right|}{\left|B_{2 n+2} B_{2 n+3}\right|} \\
& \leqq \frac{\left|a_{2 n+3}\right|}{g_{1} g_{2} r_{1} r_{2} \cdots r_{2 n}}\left\{1-\frac{\left|a_{2 n+2}\right|}{r_{2 n+1}}\left|\frac{B_{2 n}}{B_{2 n+2}}\right|\right\}\left\{1-\frac{\left|a_{2 n+3}\right|}{r_{2 n+2}}\left|\frac{B_{2 n+1}}{B_{2 n+3}}\right|\right\} \\
& <\frac{\left|a_{2 n+3}\right|}{g_{1} g_{2} r_{1} r_{2} \cdots r_{2 n}},
\end{aligned}
$$

and, similarly,

$$
\left|\frac{A_{2 n+2}}{B_{2 n+2}}-\frac{A_{2 n+1}}{B_{2 n+1}}\right|<\frac{\left|a_{2 n+2}\right|}{g_{1} g_{2} r_{1} r_{2} \cdots r_{2 n-1}} .
$$

Now if $\lim \inf \left(r_{1} r_{2} \cdots r_{n}\right)=0$, (1.1) converges by Theorem C. If $r_{1} r_{2} \cdots r_{n}$ is bounded away from 0 , these inequalities show that if $\lim \inf \left|a_{n}\right|=0$ the continued fraction converges. Hence we have proved

Theorem 3.4. If in the continued fraction (1.1) the inequalities (1.3) hold with inequality for $n=1,2$, then the continued fraction converges if $\lim \inf \left|a_{n}\right|$ $=0$.

4. Convergence theorems involving $\sum\left|b_{n}\right|$. If the elements $a_{n}$ are different from 0 , the continued fraction (1.1) can be thrown into the form $1 / b_{1}+1 / b_{2}+1 / b_{3}+\cdots$, where $b_{1}=1, a_{n}=1 / b_{n} b_{n-1}, n=2,3,4, \cdots$. Therefore, by a theorem of von Koch, ${ }^{*}$ the even and odd approximants of the continued fraction will have distinct limits, finite or infinite, if the series $\sum\left|b_{n}\right|$ converges. Then a necessary condition for the convergence of the continued fraction is the divergence of the series $\sum\left|b_{n}\right|$.

In this section we shall give some conditions under which the divergence of the series $\sum\left|b_{n}\right|$ is sufficient to insure the convergence of the continued fraction.

TheORem D. If there exist positive numbers $r_{n}$ satisfying the inequalities (1.3), with actual inequality holding for at least one even and one odd index, and if

$$
r_{1} r_{3} r_{5} \cdots r_{2 n-1}<M, \quad r_{2} r_{4} r_{6} \cdots r_{2 n}<M
$$

\footnotetext{
* Perron, p. 235, Theorem 6.
} 
$n=1,2,3, \cdots$, where $M$ is a finite constant, then a necessary and sufficient condition for the convergence of the continued fraction

$$
1 / 1+a_{2} / 1+a_{3} / 1+a_{4} / 1+\cdots, \quad a_{n} \neq 0,
$$

is that the series $\sum\left|b_{n}\right|$ diverge, where $b_{1}=1, a_{n}=1 / b_{n-1} b_{n}, n=2,3,4, \cdots$.

Proof. We may assume that actual inequality holds in (1.3) for $n=1,2$. For if inequality holds for a certain index $n$, we may slightly increase $r_{n-2}, r_{n-4}, \cdots$ in such a way as to introduce inequality for $n=1,2$. This cannot affect condition (4.1) since the values of only a finite number of the $r_{n}$ have been changed.

In terms of the $b_{n}$ the continued fraction (4.2) takes the form

$$
1 / b_{1}+1 / b_{2}+1 / b_{3}+\cdots .
$$

If $P_{n} / Q_{n}$ is the $n$th approximant of (4.3) one may easily verify the relation

$$
b_{n+1} Q_{n+3}=\left(b_{n+1} b_{n+2} b_{n+3}+b_{n+1}+b_{n+3}\right) Q_{n+1}-b_{n+3} Q_{n-1},
$$

$n=1,2,3, \cdots$. From this we obtain by means of (1.3) the inequality

$$
\left|Q_{n+3}\right|-\frac{1}{r_{n+2}}\left|Q_{n+1}\right| \geqq r_{n}\left|\frac{b_{n+3}}{b_{n+1}}\right|\left(\left|Q_{n+1}\right|-\frac{1}{r_{n}}\left|Q_{n-1}\right|\right),
$$

$n=1,2,3, \cdots$. Since $(1.3)$ holds with actual inequality for $n=1,2$, the quantities

$$
e_{1}=\frac{\left|Q_{2}\right| r_{1}-\left|Q_{0}\right|}{\left|b_{2}\right| r_{1}}, \quad e_{2}=\frac{\left|Q_{3}\right| r_{2}-\left|Q_{1}\right|}{\left|b_{3}\right| r_{2}}
$$

are positive. On introducing the proper factors in (4.5) and summing we get

$$
r_{3} r_{5} \cdots r_{2 n+1}\left|Q_{2 n+2}\right| \geqq e_{1}\left(1+\sum_{k=1}^{n} r_{1} r_{3}^{2} r_{5}^{2} \cdots r_{2 k-1}^{2} r_{2 k+1}\left|b_{2 k+2}\right|\right)
$$

and

$$
r_{4} r_{6} \cdots r_{2 n+2}\left|Q_{2 n+3}\right| \geqq e_{2}\left(1+\sum_{k=1}^{n+1} r_{2} r_{4}^{2} r_{6}^{2} \cdots r_{2 k-2}^{2} r_{2 k}\left|b_{2 k+1}\right|\right)
$$

Now

$$
\left|\frac{P_{n}}{Q_{n}}-\frac{P_{n+1}}{Q_{n+1}}\right|=\frac{1}{\left|Q_{n} Q_{n+1}\right|} ;
$$

and the limits $\lim _{n=\infty}\left(P_{2 n} / Q_{2 n}\right), \lim _{n=\infty}\left(P_{2 n-1} / Q_{2 n-1}\right)$ both exist and are finite. Hence the continued fraction will converge if $1 /\left|Q_{n} Q_{n+1}\right|$ has the lower limit 0. By Theorem C, if $r_{1} r_{2} \cdots r_{n}$ has the lower limit 0 , our theorem is 
granted. Hence there remains only the case where, for a constant $c>0$,

$$
c \leqq r_{3} r_{5} \cdots r_{2 n-1}<M, \quad c \leqq r_{4} r_{6} \cdots r_{2 n}<M .
$$

The theorem now follows from a consideration of (4.6), (4.7), and the hypothesis that the series $\sum\left|b_{n}\right|$ is divergent.

TheOREM E. If the numbers $a_{n}$ in (1.1) are all less in absolute value than a constant $M$, and if there exist positive numbers $r_{n}$ satisfying the inequalities (1.3) with actual inequality for an even and an odd index, then the continued fraction converges.

Proof. We may suppose the $a_{n} \neq 0$, for this case is treated in Theorem A. Furthermore we may suppose that $\lim \inf \left(r_{1} r_{2} \cdots r_{n}\right) \geqq c>0$, for otherwise Theorem $\mathrm{C}$ applies. From a composition of corresponding terms in the inequalities (4.6), (4.7) we get

$$
r_{3} r_{4} \cdots r_{n+1}\left|Q_{n+1} Q_{n+2}\right| \geqq e_{1} e_{2}\left(1+\sum r_{1} r_{2} r_{3}^{2} r_{4}^{2} \cdots r_{n-1}^{2} r_{n} r_{n+1}\left|b_{n+1} b_{n+2}\right|\right) .
$$

If $\lim \sup \left(r_{1} r_{2} \cdots r_{n}\right) \leqq N$, where $N$ is finite, then

$$
\lim \sup \left|Q_{n+1} Q_{n+2}\right| \geqq \frac{e_{1} e_{2}}{N}\left(r_{1} r_{2}+\sum_{k=1}^{\infty} r_{1}^{2} r_{2}^{2} \cdots r_{k-1}^{2} r_{k} r_{k+1} M\right),
$$

and it is evident that the series on the right diverges. Then we have $\lim \sup \left|Q_{n+1} Q_{n+2}\right|=\infty$. If $N$ is infinite we have $\left|Q_{n+1} Q_{n+2}\right|>e_{1} e_{2}\left(r_{1} r_{2} \cdots r_{n+1}\right) M$, and $\lim \sup \left|Q_{n+1} Q_{n+2}\right|=\infty$. The theorem now follows from (4.8).

5. Convergence regions. By a convergence region for the continued fraction (1.1) we shall understand a set $W$ of points in the complex plane such that if $a_{2}, a_{3}, a_{4}, \cdots$ are arbitrary points in $W$ the continued fraction converges. A convergence region is necessarily bounded. For if $W$ were unbounded, we could choose the $a_{n} \neq 0$ in $W$ in such a way that the series $\sum\left|b_{n}\right|$ would be convergent, where $b_{1}=1, a_{n}=1 / b_{n-1} b_{n}$, and for this choice of the $a_{n}$ the continued fraction would diverge by oscillation. On the other hand, if the $a_{n} \neq 0$ lie in any bounded region, it is clear that the series $\sum\left|b_{n}\right|$ must diverge.

Worpitzky* showed that the interior and boundary of the circle $|z|=1 / 4$ is a convergence region for (1.1); and since (1.1) diverges if $a_{n}=-1 / 4-c$ where $c$ is real and positive, it follows that the interior and boundary of a circle with center at the origin and radius greater than $1 / 4$ cannot be a convergence region. Szász $†$ obtained convergence regions containing the origin, which neither contain nor are contained in the circle $|z|=1 / 4$.

* See the second footnote on page 156.

† Szász, Journal für die reine und angewandte Mathematik, vol. 147 (1916), pp. 132-160. 
We now propose to characterize completely all convergence regions which are symmetric with respect to the real axis.

In order to obtain a necessary restriction upon such a region, consider the continued fraction $1 / 1+\bar{z} / 1+z / 1+\bar{z} / 1+z / 1+\cdots$, in which $z=x+i y$, $\bar{z}=x-i y, x, y$ real. If this continued fraction converges, then the continued fraction

$$
-z+\frac{z}{1}+\frac{\bar{z}}{1}+\frac{z}{1}+\frac{\bar{z}}{1}+\frac{z}{1}+\cdots
$$

must converge, as well as the continued fraction

$$
\frac{\left|z^{2}\right|}{1+2 x}-\frac{\left|z^{2}\right|}{1+2 x}-\frac{\left|z^{2}\right|}{1+2 x}-\cdots,
$$

having as approximants the odd approximants of (5.1). Now if the latter converges, it must have a real value, which is a root of the quadratic equation in $u$ :

$$
u^{2}-(1+2 x) u+\left(x^{2}+y^{2}\right)=0 .
$$

Consequently, it is necessary for the convergence of (5.1) that $y^{2} \leqq x+1 / 4$, that is, $z$ shall lie within or upon the parabola $|z|-\Re(z)=1 / 2$, where $\Re(z)$ denotes the real part of $z$. Therefore a convergence region for (1.1) which is symmetric with respect to the real axis is necessarily a finite region bounded by this parabola. We shall prove that this necessary condition is also a sufficient condition.

TheOREM F. (The parabola theorem.) If the elements $a_{n}$ of the continued fraction (1.1) lie within or upon the parabola (see figure)

$$
|z|-\Re(z)=1 / 2 \text {, }
$$

then:

(a) The denominator of the nth approximant $A_{n} / B_{n}$ is different from 0 for all $n$.

(b) The sequences of even and odd approximants have finite limits $L_{0}$ and $L_{1}$, and $\left|L_{0}-L_{1}\right| \leqq 1$.

(c) If $a_{n} \neq 0, n=2,3,4, \cdots$, the continued fraction converges if and only if the series $\sum\left|b_{n}\right|$ diverges, where $b_{1}=1, a_{n}=1 / b_{n} b_{n-1}, n=2,3,4, \cdots$.

(d) If some $a_{n}$ vanishes, the continued fraction converges and equals one of its approximants.

(e) The parabola (5.2) is the best possible curve symmetric with respect to the real axis having these properties. 
Proof. Part (e) follows from the fact shown above that when $a_{2 n}=\bar{z}$, $a_{2 n-1}=z$, the continued fraction diverges if $z$ lies outside the parabola.

The other parts of the theorem will follow from Theorems A and D, if we show that the inequalities (1.3) hold for $r_{n}=1$, with actual inequality for $n=1,2$.

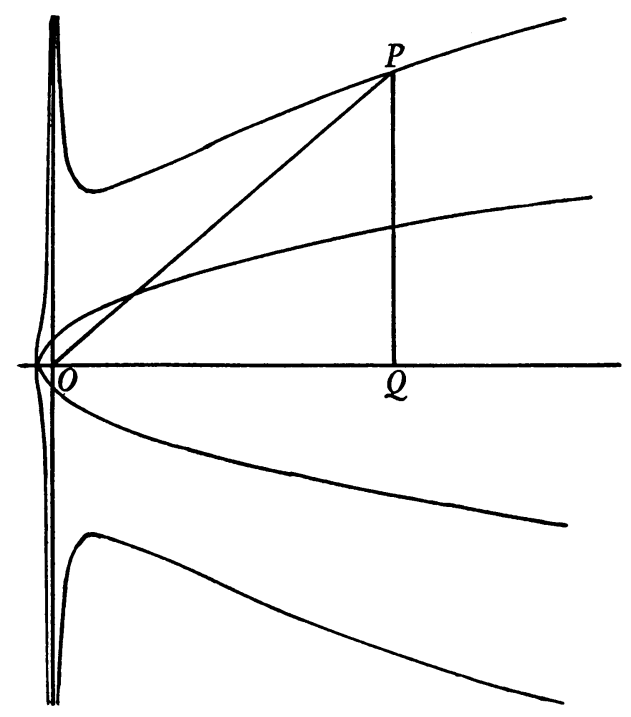

Put $a_{n}=u_{n}+i v_{n}$ where $u_{n}, v_{n}$ are real. Then if $a_{n}$ lies in or upon the parabola (5.2), we must have

$$
\left|a_{n}\right|=u_{n}+h_{n} / 2, \quad n=2,3,4, \cdots,
$$

where $0 \leqq h_{n} \leqq 1$. We then have

$$
\begin{aligned}
\left|1+a_{2}\right| & \geqq 1+u_{2}>u_{2}+h_{2} / 2=\left|a_{2}\right|, \\
\left|1+a_{2}+a_{3}\right| & \geqq 1+u_{2}+u_{3}=\left(1-h_{2} / 2-h_{3} / 2\right)+\left|a_{2}\right|+\left|a_{3}\right|>\left|a_{3}\right|, \\
\left|1+a_{n}+a_{n+1}\right| & \geqq 1+u_{n}+u_{n+1} \geqq u_{n}+h_{n} / 2+u_{n+1}+h_{n+1} / 2 \\
& \geqq\left|a_{n}\right|+\left|a_{n+1}\right|,
\end{aligned}
$$

$n=3,4,5, \cdots$, as was to be proved.

It is of interest to note the analogy between the above result and the following important and general convergence criterion of Van Vleck:*

THEOREM 5.1. (Van Vleck.) Let $b_{n}=\left|b_{n}\right| e^{i \theta_{n}}$ where

$$
-\pi / 2+\epsilon \leqq \theta_{n} \leqq \pi / 2-\epsilon, \quad \epsilon>0,
$$

* Perron, pp. 264-271. 
$n=1,2,3, \cdots$, and suppose not all the $b_{n}$ with odd index are 0. The then sequences of even and odd approximants of the continued fraction

$$
1 / b_{1}+1 / b_{2}+1 / b_{3}+\cdots
$$

have finite limits; and the continued fraction converges if and only if the series $\sum\left|b_{n}\right|$ diverges.

It is interesting to note that the expression $\left|a_{n}\right|-\Re\left(a_{n}\right)$ appearing in the parabola theorem occurs in the following theorem of Szász:*

THEOREM 5.2. (Szász.) The continued fraction (1.1) converges if the series $\sum\left|a_{n}\right|$ converges, and

$$
\sum_{2}^{\infty}\left\{\left|a_{n}\right|-\Re\left(a_{n}\right)\right\}<2 .
$$

It is easy to construct examples where convergence can be established by Theorem 5.1 but not by Theorem A or our later theorems, and vice versa. The same statement applies to Theorem 5.2, where, however, at most three of the $a_{n}$ 's can lie outside the parabola. But if, for example, $a_{2}$ is taken outside the parabola equal to $-1+\epsilon$, and $a_{3}=a_{4}=1$, then if the other $a_{n}$ 's are chosen so that

$$
\sum_{5}^{\infty}\left|a_{n}\right|-\sum_{5}^{\infty} \Re\left(a_{n}\right)<2 \epsilon
$$$$
\epsilon>0
$$

the conditions of Szász are satisfied, but the inequalities (1.3) cannot hold for nonnegative $r_{n}$. In fact, we must have $r_{1} \geqq\left|a_{2}\right| /\left|1+a_{2}\right|=1 / \epsilon-1$. But $\left|1+a_{3}+a_{4}\right|<r_{1}\left|a_{3}\right|$ if $\epsilon<1 / 4$, and hence it is not possible to determine $r_{3} \geqq 0$.

We have seen that a convergence region for (1.1) which is symmetric with respect to the real axis must lie in the parabola (5.2). We now turn our attention to the problem of finding convergence regions which are not symmetric with respect to the real axis.

Theorem G. (The triangle theorem.) Let $P(x, y)$ be any point upon the curve (see figure after Theorem $\mathrm{F}$ )

$$
y=(2 x+1)(4 x+1)^{1 / 2} / 2 x,
$$

in the plane of $z=x+i y$. Let $O$ denote the origin and $Q$ the point $z=x$. Then the closed region made up of the interior and boundary of the triangle $O P Q$ is a convergence region for the continued fraction (1.1).

Proof. As in the proof of Theorem F, we show that Theorem D applies

\footnotetext{
* Perron, p. 259.
} 
with $r_{n}=1$. Let $m$ be the slope of the line $O P$. Then if $a_{n}$ is in the triangle $O P Q$, we may write $a_{n}=u_{n}\left(1+i m t_{n}\right)$, where $u_{n}$ is the real part of $a_{n}$, and $0 \leqq t_{n} \leqq 1$. Since $u_{2}>-1 / 2$ it follows that $\left|1+a_{2}\right|>\left|a_{2}\right|$, so that the first inequality (1.3) holds with $r_{1}=1$. Since $u_{n} \geqq-1 / 4, u_{2} u_{3} \geqq 0, v_{2} v_{3} \geqq 0$, where $v_{n} i$ is the imaginary part of $a_{n}$, it is easily seen that $\left|1+a_{2}+a_{3}\right|>\left|a_{3}\right|$, so that the second inequality (1.3) holds. Thus actual inequality holds in the first two relations (1.3). The remaining inequalities will hold provided

$$
\begin{aligned}
\left(1+2 u_{n}+2 u_{n+1}+\right. & \left.2 u_{n} u_{n+1}\right) \\
& \geqq 2 u_{n} u_{n+1}\left[\left(1+m^{2} s^{2}\right)^{1 / 2}\left(1+m^{2} t^{2}\right)^{1 / 2}-2 m^{2} s^{2} t^{2}\right],
\end{aligned}
$$

$n=3,4,5, \cdots$, where $s=t_{n}, t=t_{n+1}$. We find that the maximum of the bracketed part in the right-hand member of (5.4) for $s, t$ between 0 and 1 is $\left(1+m^{2}\right)^{1 / 2}$. Hence (5.4) will hold if

$$
\left[\left(1+m^{2}\right)^{1 / 2}-1\right] u_{n} u_{n+1}-u_{n}-u_{n+1} \leqq 1 / 2 .
$$

We next maximize the left-hand member of (5.5) in the two cases $u_{n}>0, u_{n}<0$, and find that in the first case (5.5) will hold if $w=u_{n}$ satisfies the inequality

$$
w \leqq\left[2\left\{\left(1+\left[1+m^{2}\right]^{1 / 2}\right)\right\}^{1 / 2}-2\right]^{-1},
$$

while in the second case (5.5) will hold provided $w=-u_{n}$ satisfies (5.6). But this is precisely the condition that the point $P$ shall lie upon the curve (5.3). The proof of Theorem $\mathrm{G}$ is now complete.

It is easy to show that any bounded set of points lying upon a ray from the origin, in the right half-plane, is a convergence region for (1.1). In fact, upon such a ray the inequalities (1.3) hold for $r_{n}=1$ with actual inequality throughout. To see this, put $a_{n}=\alpha_{n} e^{2 i \theta_{n}}$ in (1.3), and the latter take the form

$$
\begin{aligned}
& \left(1+\alpha_{n}+\alpha_{n+1}\right)^{2}-\left(r_{n-2} \alpha_{n}+\frac{\alpha_{n+1}}{r_{n}}\right)^{2} \\
& \quad \geqq 4\left\{\alpha_{n} \sin ^{2} \theta_{n}+\alpha_{n} \alpha_{n+1} \sin ^{2}\left(\theta_{n+1}-\theta_{n}\right)+\alpha_{n+1} \sin ^{2} \theta_{n+1}\right\},
\end{aligned}
$$

$n=1,2,3, \cdots,\left(r_{0}=r_{-1}=\alpha_{1}=0\right)$. When $r_{n}=1, \theta_{n}=\theta=$ const., these reduce to

$$
\begin{aligned}
1+2 \alpha_{2} \geqq 0, \quad\left(\alpha_{2}+\alpha_{3}\right) \cos 2 \theta & \geqq-\left(1+\alpha_{2}{ }^{2}\right) /\left(\alpha_{2}+\alpha_{3}\right), \\
\left(\alpha_{n}+\alpha_{n+1}\right) \cos 2 \theta & \geqq-1 / 2,
\end{aligned}
$$

$n=3,4,5, \cdots$. These are obviously satisfied with actual inequality throughout, if $-\pi / 2 \leqq 2 \theta \leqq \pi / 2$.

As an application, consider an arbitrary sequence $x_{1}, x_{2}, x_{3}, \cdots$ with nonnegative real parts. Let $v_{n}$ be the ray from the origin upon which $x_{n}$ lies. 
Choose $y_{n}$ upon $v_{n}$ but in the parabola (5.2). Then if $a_{2}=x_{1}, a_{3}=y_{1}, a_{4}=y_{2}$, $a_{5}=x_{2}, a_{6}=y_{2}, a_{7}=y_{3}, a_{8}=x_{3}, \cdots$, it is clear that the inequalities (1.3) are satisfied with $r_{n}=1$. If, in particular, the $y_{n}$ have a limit point in the finite plane (and they can evidently be so chosen), then the continued fraction $1 / 1+a_{2} / 1+a_{3} / 1+\cdots$ converges by Theorem $\mathrm{D}$. This shows that it is possible for the $a_{n}$ to be everywhere dense in the right half-plane for a convergent continued fraction (1.1).*

6. The problem of Szász.† Szász proposed the following general problem: To assign neighborhoods to the elements of a given convergent continued fraction in which these elements may vary without destroying the convergence property of the continued fraction. We shall indicate in this section how Theorem A may be applied to this problem.

We consider a convergent continued fraction

$$
\frac{1}{1}+\frac{c_{2}}{1}+\frac{c_{3}}{1}+\cdots
$$

and seek to determine positive numbers $t_{2}, t_{3}, t_{4}, \cdots$ such that if

$$
\left|a_{n}-c_{n}\right| \leqq t_{n}, \quad n=2,3,4, \cdots,
$$

then the continued fraction

$$
\frac{1}{1}+\frac{a_{2}}{1}+\frac{a_{3}}{1}+\cdots
$$

will converge. The theorems of the preceding sections may be applied to this problem in certain cases. For that purpose put

$$
a_{n}=c_{n}+\delta_{n} t_{n}, \quad n=2,3,4, \cdots, 0 \leqq\left|\delta_{n}\right| \leqq 1,
$$

in the inequalities (1.3). Then these inequalities will hold provided

$$
\begin{aligned}
r_{1}\left|1+c_{2}\right| & \geqq\left|c_{2}\right|+\left(1+r_{1}\right) t_{2}, \\
r_{2}\left|1+c_{2}+c_{3}\right| & \geqq\left|c_{3}\right|+\left(1+r_{2}\right) t_{3}+r_{2} t_{2}, \\
r_{n}\left|1+c_{n}+c_{n+1}\right| & \geqq r_{n} r_{n-2}\left|c_{n}\right|+\left|c_{n+1}\right|+\left(1+r_{n}\right) t_{n+1}+r_{n}\left(1+r_{n-2}\right) t_{n},
\end{aligned}
$$

$n=3,4,5, \cdots$. It follows that if there exist positive numbers $r_{n}, t_{n}$ satisfying (6.3), then

$$
r_{n}\left|1+c_{n}+c_{n+1}\right| \geqq r_{n} r_{n-2}\left|c_{n}\right|+\left|c_{n+1}\right|,
$$

$n=1,2,3, \cdots,\left(r_{-1}=r_{0}=a_{1}=0\right)$, and hence under suitable restrictions, (1.1)

* Leighton and Wall showed that this can be done for the entire plane. See American Journal of Mathematics, vol. 58 (1936), pp. 267-281; p. 269.

† See the second footnote on page 165 . 
will converge if (6.2) holds. If the $a_{n}, c_{n}, t_{n}$ are functions of variables, and the $r_{n}$ are independent of the variables, then under suitable conditions the convergence will be uniform. Put

It is convenient to specialize the inequalities (6.3) in the following way.

$$
\begin{aligned}
& s_{1}=\frac{r_{1}\left|1+c_{2}\right|-\left|c_{2}\right|}{1+r_{1}}, \quad s_{2}=\frac{r_{2}\left|1+c_{2}+c_{3}\right|-\left|c_{3}\right|}{1+2 r_{2}} \\
& s_{n}=\frac{r_{n}\left|1+c_{n}+c_{n+1}\right|-r_{n} r_{n-2}\left|c_{n}\right|-\left|c_{n+1}\right|}{1+2 r_{n}+r_{n} r_{n-2}}
\end{aligned}
$$

$n=3,4,5, \cdots$. If $s_{1}, s_{2}, s_{3}, \cdots \geqq 0$, and $t_{n}^{\prime}$ is the larger of $s_{n-1}, s_{n}$, then $t_{n}=t_{n}{ }^{\prime},(n \geqq 2)$, satisfy the inequalities (6.3). We therefore have this theorem:

THEOREM 6.1. Let $a_{2}, a_{3}, a_{4}, \cdots, c_{2}, c_{3}, c_{4}, \cdots$ be functions of any variables over a domain $D$, and suppose that there exist numbers $r_{1}, r_{2}, r_{3}, \cdots$, nonnegative and independent of the variables such that the quantities $s_{n}$, defined by (6.5), are nonnegative over $D$. Then if

$$
\left|a_{n}-c_{n}\right| \leqq\left\{\begin{array}{l}
s_{n-1} \\
s_{n}
\end{array}, \quad n=2,3,4, \cdots,\right.
$$

we shall have $r_{n}\left|1+a_{n}+a_{n+1}\right| \geqq r_{n} r_{n-2}\left|a_{n}\right|+\left|a_{n+1}\right|$ for $n=1,2,3, \cdots$, $\left(r_{-1}=r_{0}=a_{1}=0\right)$. Hence, in particular, if $1+\sum r_{1} r_{2} \cdots r_{n}$ converges, the continued fraction (1.1) will converge uniformly over $D$.

As an illustration, let $c_{n}=(2 n-3) / 4, n \geqq 2 ; r_{n}=n /(n+2), n \geqq 1$. Then $s_{1}=1 / 8, s_{n}=(n-1) /(4 n), n \geqq 2$, and hence the continued fraction (1.1) in which $a_{2}=\left(1+4 x_{2}\right) / 4, a_{3}=\left(3+4 x_{3}\right) / 4, \cdots, a_{n}=\left(2 n-3+4 x_{n}\right) / 4, \cdots$ converges uniformly for

$$
\left|x_{2}\right| \leqq 1 / 8, \quad\left|x_{n}\right| \leqq 1 / 4-1 /[4(n-1)],
$$

$n=3,4,5, \cdots$.

If $c_{n}=c$ and $\Re(c)>-1 / 4$, we can determine by means of Theorem 6.1 a neighborhood of $c$ which is a convergence region for (1.1).

TheOREM 6.2. Let $c, a_{2}, a_{3}, a_{4}, \cdots$ be functions of any variables over $a$ certain domain $D$ in which

$$
\left|a_{n}-c\right| \leqq \frac{|1+2 c|-2|c|}{4}, \quad n=2,3,4, \cdots .
$$

Then the continued fraction (1.1) converges uniformly over $D$. 
Proof. Take $r_{n}=1, c_{n}=c$ in Theorem 6.1. Then the theorem follows at once by application of Theorem $\mathrm{D}$.

It is not difficult to show by means of Theorem $\mathrm{A}$, with $r_{n}=n /(n+2)$, that 2 is the least upper bound of the absolute value of the continued fraction in Theorem 6.2.

Theorem 6.2 gives a generalization of the Worpitzky theorem, which reduces to the latter when $c=0$. It is applicable whenever the real part of $c$ exceeds $-1 / 4$, and in this case furnishes a solution of the problem of Szász for the periodic continued fraction $1 / 1+c / 1+c / 1+\cdots$. Szász obtained a solution except when $c$ is real and not greater than $-1 / 4$, in which case there is no solution. We note that when $c=1$, the theorem of Szász gives as the radius of the convergence circle approximately $5 / 22$, Theorem 6.1 gives the value $1 / 4$, and the parabola theorem gives the value 1 . Moreover, from the latter theorem it follows that the radius of a convergence circle with center $c=1$ cannot exceed 1 .

Armour Institute of Technology, Chicago, Ill.,

NORTHWESTERN UNIVERSITY, Evanston, Ill. 